\title{
An Efficient Geometric Integrator for Thermostatted Anti-/Ferromagnetic Models
}

\author{
Teijo Arponen \\ Institute of Mathematics \\ Helsinki University of Technology \\ Finland
}

\author{
Ben Leimkuhler \\ Department of Mathematics \\ University of Leicester \\ U.K.
}

5th November 2018

\begin{abstract}
(Anti)-/ferromagnetic Heisenberg spin models arise from discretization of LandauLifshitz models in micromagnetic modelling. In many applications it is essential to study the behavior of the system at a fixed temperature. A formulation for thermostatted spin dynamics was given by Bulgac and Kusnetsov [5], which incorporates a complicated nonlinear dissipation/driving term while preserving spin length. It is essential to properly model this term in simulation, and simplified schemes give poor numerical performance, e.g. requiring an excessively small timestep for stable integration. In this paper we present an efficient, structure-preserving method for thermostatted spin dynamics.
\end{abstract}

Keywords: Heisenberg ferromagnet, micromagnetics, spin dynamics, Landau-Lifschitz equation, Gilbert damping, thermostats, constant temperature, domain walls, geometric integrator, reversible method

\section{Introduction}

In recent years geometric integrators have become ubiquitous for numerical treatment of differential equations. By a geometric integrator is meant a numerical method that preserves some known structure of the continuous flow. Geometric integrators are particularly important for long term simulations, as used in molecular sampling or celestial mechanics. In this paper we consider the application of geometric integration principles for the types of spin dynamics systems which arise frequently in modelling of ferromagnets and antiferromagnets. Efficient Lie-Poisson schemes for classical spin dynamics described by the Landau-Lifshitz (LL) equation were studied in [10], and related multisymplectic schemes in [9]. Here we develop and test a geometric integrator for a semi-discrete Landau-LifshitzGilbert (LLG) equation which includes a nonlinear dissipative term. This dissipative system forms the foundation for a more complicated thermostatted model, following the approach of Bulgac and Kusnetsov [5, 2, We design an effective splitting technique for the full coupled system.

LL and LLG are currently a very active topic of research. Other approaches to them can be found in [9, 23, 6, 10, 19, 21, 14, 17, who also provide further references. However none of these consider a thermostatted version. 
Simulation with the thermostatted version shows fascinating global behavior: the system first arranges into patterns (spin domains) with slowly moving domain walls, then goes into a quasi-chaotic state and quickly rearranges itself into completely new spin domains. This kind of transition would not be possible with local interactions only. Here the thermostatting variable is defined in such a way that it has a global character.

A number of recent articles have focussed on the geometric integration of molecular systems in the canonical ensemble [4, 15, 3, 12. In these articles, the aim has been to start from a Hamiltonian formulation for thermostatted molecular simulation and then to provide a suitable symplectic integrator. The starting point is usually Nosé dynamics, although generalizations are possible.

Since the constant energy Heisenberg spin system is Lie-Poisson, it is natural to seek a Lie-Poisson system to model the action of the thermostat. While it is possible (with some additional complication, due to the presence of constraints) to develop such a model for the thermostatted Heisenberg model, based on the ideas in [12, it is much different in character from the corresponding molecular dynamics models (see the appendix). In particular, this approach appears to require introduction of many thermostatting variables which act differently on each spin vector of the system. In the context of magnetic models, this approach therefore sacrifices an important feature of Nosé molecular dynamics: the apparent compatibility between the thermostatted quasi-dynamics and the microcanonical dynamics. (Even though Nosé dynamics is typically only validated based on a phase-sampling correspondence, there is widespread agreement that the thermostatted dynamics is relevant for modelling dynamics of an appropriate extended system which is not too different in character from the microcanonical version.) Moreover, the Lie-Poisson thermostats add additional complexity in the form of a relatively complex bath model.

Given these complications, we believe the best available starting point for geometric integration of thermostatted spin dynamics is the alternative framework of Bulgac and Kusnetsov, based loosely on Nosé-Hoover (NH) dynamics. Like NH molecular dynamics, these formulations sacrifice Hamiltonian structure, while retaining a reversing symmetry. It is unclear the extent to which this loss of structure affects the stability of methods and the ultimate resolution of macroscopic features of the spin model. Although in molecular dynamics it is known that the reversible-only methods are often inferior to their symplectic counterparts [13, it is also well established that NH-type methods are far superior to methods that are neither symplectic nor reversible.

The rest of the paper is organized as follows: in Section 1.1 we review splitting methods and apply them to our models. In Sections 2, 4 we present the models and methods in detail. In Section 5 we present numerical results. Finally in Section 6 we present some conclusions and discussion.

\subsection{Background: Review of splitting methods}

The reader is referred to [16, 18, for a detailed discussion of splitting methods. To briefly describe their basic construction, consider a differential equation $\dot{u}=f(u)$, with flow map $\Phi_{\tau, f}$. If $f=f_{1}+f_{2}$, we have $\Phi_{\tau, f}=\Phi_{\tau, f_{1}} \circ \Phi_{\tau, f_{2}}+O\left(\tau^{2}\right)$. If the flows on vector fields $f$, $f_{1}$, and $f_{2}$ share a first integral, then the composed map will preserve it as well. In this way, geometric integrators can be developed to preserve general classes of Lie groups. If the vector field is time-reversible, i.e. $f(R u)=-R f(u)$ for some linear involution $R$, then the symmetric concatenation or "Strang Splitting" $\hat{\Phi}_{\tau, f}=\Phi_{\frac{1}{2} \tau, f_{1}} \circ \Phi_{\tau, f_{2}} \circ \Phi_{\frac{1}{2} \tau, f_{1}}$, where $f_{1}, f_{2}$ are reversible vector fields, gives a time-reversible map $\left(R \hat{\Phi}_{\tau, f}^{-1}=\hat{\Phi}_{\tau, f} \circ R\right)$, which, moreover, provides a second-order approximation of the solution on a finite time interval. 
As an example, if $H=H(q, p)=T(p)+V(q)$, the leapfrog (Störmer/Verlet) integrator results from the concatenation $\hat{\Phi}_{\tau, H}=\Phi_{\frac{1}{2} \tau, V} \circ \Phi_{\tau, T} \circ \Phi_{\frac{1}{2} \tau, V}$.

The construction of splitting methods for various types of flows, and with various orders of accuracy, is discussed in a number of papers (see, e.g, 24, 20]). Practical splitting-based geometric integrators have been constructed by mathematicians, chemists and physicists for a wide variety of important applications, including the rigid body, general holonomic constraints, particle accelerator models, and the solar system. Vector field splittings were used in [10] to obtain efficient time-reversible integrators for (undamped) spin systems; it is this fundamental scheme that we have extended in this paper to treatment of dissipative and thermostatted systems.

\section{The original Landau-Lifshitz model as a Poisson system}

There are several versions of the Landau-Lifshitz equation depending on which forces and fields are taken into account. The version we use here is that of [7], discarding the external and demagnetizing field. (Schemes for more general formulations would build on the work presented here.) The equation can be written in the form:

$$
\frac{\partial}{\partial t} S=S \times \nabla^{2} S+S \times D S
$$

where $x \in I \times I \subset \mathbb{R}^{2}, I$ an interval, $S(x, t)$ is a unit vector in $\mathbb{R}^{3}$ representing the classical spin at position $x$ and time $t$, and $D$ is a diagonal matrix representing anisotropy. Clearly $|S|=$ constant in time:

$$
\frac{\partial}{\partial t}|S(x, t)|^{2}=2 S(x, t) \cdot \frac{\partial}{\partial t} S(x, t)=0 \quad \forall x, t .
$$

Following the usual practice, we discretize the spatial variable $x$ using second order central differences on a regular lattice as in [10] so that in the discretized system the unit length property is conserved. We then get a Poisson system on a lattice. Without loss of generality we may assume the lattice size to be 1:

$$
\begin{aligned}
S(x, \cdot) & \mapsto z_{i j} \\
\nabla^{2} S(x, \cdot) & \mapsto z_{i, j-1}+z_{i, j+1}+z_{i-1, j}+z_{i+1, j}-4 z_{i j}
\end{aligned}
$$

hence (11) becomes

$$
\dot{z}_{i j}=z_{i j} \times\left(z_{i, j-1}+z_{i, j+1}+z_{i-1, j}+z_{i+1, j}-4 z_{i j}\right)+z_{i j} \times D z_{i j}
$$

Note that the $-4 z_{i j}$ term can be dropped out. Here we have an $n \times n$ lattice of spins: the variable $z_{i j}$ is on the unit sphere of $\mathbb{R}^{3}$ when $i, j \in\{1, \ldots, n\}$. When either $i$ or $j$ index is zero or $n+1$, those represent boundaries. Except for the case of periodic boundary conditions, these boundary terms are different from the spins: they are an artefact of discretization, and do not have a counterpart in the continuum case (11). Especially, they are not necessarily of unit length. We do not represent equations of motion to them, hence they are assumed constants.

By periodic boundary conditions we mean

$$
z_{0 j}=z_{n j}, \quad z_{i 0}=z_{i n}
$$


Next we define the Poisson structure matrix. Let us denote

$$
z:=\left[z_{11}^{T}\left|z_{12}^{T}\right| \ldots\left|z_{1 n}^{T}\right| z_{21}^{T}\left|z_{22}^{T}\right| \ldots \mid z_{n n}^{T}\right]^{T},
$$

i.e. $z$ is a column vector. For an arbitrary $v=:[a, b, c]^{T} \in \mathbb{R}^{3}$ we denote

$$
\hat{v}:=\left(\begin{array}{ccc}
0 & -c & b \\
c & 0 & -a \\
-b & a & 0
\end{array}\right), \quad \hat{v} u=v \times u \quad \forall u .
$$

The Poisson structure matrix is defined as the block diagonal

$$
J(z):=\left(\begin{array}{ccccc}
\hat{z}_{11} & & & & \\
& \hat{z}_{12} & & & \\
& & \hat{z}_{13} & & \\
& & & \ddots & \\
& & & & \hat{z}_{n n}
\end{array}\right) .
$$

Now (21) becomes

$$
\dot{z}=J(z) \nabla H(z),
$$

when we choose the Hamiltonian $H$

$$
H:=-\frac{1}{2}\left(\sum_{i, j} \sum_{(a, b) \in N N(i j)} z_{i j} \cdot z_{a b}+\sum_{i, j} z_{i j}^{T} D z_{i j}+H_{0}\right),
$$

where NN refers to "nearest neighbours":

$$
N N(i j)=\left\{z_{i, j-1}, z_{i, j+1}, z_{i-1, j}, z_{i+1, j}\right\},
$$

and $H_{0}$ represents the boundaries. For example, if we have zero boundaries $\left(z_{i 0}=0, z_{0 j}=\right.$ $0, z_{i, n+1}=0, z_{n+1, j}=0$ ), then

$$
H_{0}:=0,
$$

while if we have periodic boundary conditions, then

$$
H_{0}:=\sum_{j} z_{0 j} \cdot z_{1 j}+\sum_{i} z_{i 0} \cdot z_{i 1} .
$$

We can easily extend this to a model covering both ferromagnet and antiferromagnet case.

$$
H:=-j_{K} \frac{1}{2}\left(\sum_{i, j} \sum_{N N} z_{i j} \cdot z_{a b}+\sum_{i, j} z_{i j}^{T} D z_{i j}+H_{0}\right),
$$

where $j_{K}$ is the so called exchange integral [1], assumed constant here, as in [2], and

$$
j_{K} \begin{cases}>0 & \text { for ferro } \\ <0 & \text { for antiferro. }\end{cases}
$$

Hence we have the Poisson system:

$$
\dot{z}=J(z) \nabla H(z), \quad H \text { as in (7). }
$$


For an individual spin at the lattice point $(i, j)$ this becomes

$$
\begin{aligned}
\dot{z}_{i j} & =-j_{K} z_{i j} \times\left(\sum_{(a, b) \in N N(i j)} z_{a b}\right)-j_{K} z_{i j} \times D z_{i j} \\
& =z_{i j} \times \nabla H(z)
\end{aligned}
$$

in both periodic and non-periodic cases. From now on we employ the notation

$$
\sum_{N N(i j)} z:=\sum_{(a, b) \in N N(i j)} z_{a b} .
$$

Lemma 2.1. Any system of the form $\dot{z}=J(z) v(z)$ with (44) and $v$ an arbitrary vector function, conserves the spin lengths in time:

$$
\left|z_{i j}(t)\right|=\left|z_{i j}(0)\right| \quad \forall i, j, t
$$

Proof.

$$
\frac{d}{d t}\left|z_{i j}\right|^{2}=2 z_{i j} \cdot \dot{z}_{i j}=2 z_{i j} \cdot z_{i j} \times v(z) \equiv 0
$$

This gives us useful freedom in modelling. Next, the anisotropy term $D S$ is approximated by an average:

$$
D z_{i j} \mapsto D \frac{1}{4}\left(z_{i, j-1}+z_{i, j+1}+z_{i-1, j}+z_{i+1, j}\right)
$$

this is sometimes referred to [10] as the Roberts discretization. Now (9) becomes

$$
\begin{aligned}
\dot{z}_{i j} & =-j_{K} z_{i j} \times M\left(z_{i, j-1}+z_{i, j+1}+z_{i-1, j}+z_{i+1, j}\right) \\
& =z_{i j} \times \nabla H(z),
\end{aligned}
$$

where $M=I+D / 4$ is a diagonal matrix and $H$ is modified according to (11).

\section{Numerical method}

As we noted above, (8) is a Lie-Poisson system whose meaning we recall here: we can define

$$
\{f, g\}(z):=\nabla f(z) \cdot(J(z) \nabla g(z))
$$

which fulfills the Jacobi identity

$$
\{\{f, g\}, h\}+\{\{g, h\}, f\}+\{\{h, f\}, g\}=0,
$$

hence $\{\cdot, \cdot\}$ is a Poisson bracket and $J$ is a Poisson structure matrix. Since $J$ is linear with respect to $z$, this Poisson structure can be derived from a Lie algebra structure, hence it is called a Lie-Poisson structure.

For a detailed discussion on how to integrate this, see [10]. To summarize that paper, the best way to integrate is to split the vector field in even-odd (or red-black) way:

$$
\dot{z}_{i j}=V_{1}+V_{2}
$$


where

$$
\begin{aligned}
& V_{1}= \begin{cases}-j_{K} z_{i j} \times M \sum_{N N(i j)} z, & i+j \text { even } \\
0, & i+j \text { odd },\end{cases} \\
& V_{2}= \begin{cases}0, & i+j \text { even } \\
-j_{K} z_{i j} \times M \sum_{N N(i j)} z, & i+j \text { odd } .\end{cases}
\end{aligned}
$$

Now, both of these flows can be explicitly solved. For example $V_{1}$ : for $i+j$ odd $z_{i j}(t)=$ $z_{i j}(0)$. For $i+j$ even, the sum over $N N(i j)$ includes only pairs $a, b$ with $a+b$ odd, hence they are constants (during $V_{1}$ ). Likewise in $V_{2}$ the sum is a constant. Denote the integrator of $V_{1}$ by $\hat{\Phi}_{1, t}$ and that of $V_{2}$ by $\hat{\Phi}_{2, t}$. That is,

$$
\Phi_{1, t}=\exp \left(t V_{1}\right), \quad \Phi_{2, t}=\exp \left(t V_{2}\right) .
$$

The implemented integrator is a symmetric composition of these exact flows:

$$
\hat{\Phi}_{t}:=\Phi_{2, \frac{t}{2}} \circ \Phi_{1, t} \circ \Phi_{2, \frac{t}{2}} .
$$

This integrator

- is time reversible

- conserves spin lengths

- in isotropic case $(D=I)$ preserves energy

since $\Phi_{1, t}$ and $\Phi_{2, t}$ do. See also Section 1.1.

\section{Dissipated version}

It is customary to add a dissipation term to (11). In our case the corresponding dissipated version is derived from (12) and becomes

$$
\dot{z}_{i j}=z_{i j} \times \nabla H(z)+\alpha z_{i j} \times z_{i j} \times \nabla H(z)
$$

where $\alpha$ is a dissipation constant and the corresponding term is known as the Gilbert damping term.

Clearly (15) can be written more compactly

$$
\dot{z}=J \nabla H+\alpha J^{2} \nabla H \text {. }
$$

From lemma 2.1] it follows that $|z|=1$ everywhere, i.e. the dissipation does not affect spin lengths. Let us first look at the Gilbert damping term more closely through the equation

$$
\dot{z}=\alpha z \times(z \times B)
$$

where $z \in \mathbb{R}^{3}$, and $\alpha \in \mathbb{R}$ and $B \in \mathbb{R}^{3}$ are constants. Or, more compactly,

$$
\dot{z}=\alpha J^{2} B
$$


This can be explicitly solved. Put

$$
\begin{aligned}
v & :=z \cdot B, \\
w & :=z \times B,
\end{aligned}
$$

then (17) is

$$
\begin{aligned}
\dot{v} & =\alpha\left(-C_{1}+v^{2}\right), \\
\dot{w} & =\alpha v w, \\
\dot{z} & =\alpha z \times w,
\end{aligned}
$$

where $C_{1}>0$ constant,

$$
C_{1}=|z|^{2}|B|^{2} .
$$

We can solve for $v$ (we have assumed $|z(0)|=1$ ):

$$
v(t)=-|B| \frac{\mathcal{E}^{2} C_{2}-1}{\mathcal{E}^{2} C_{2}+1},
$$

where

$$
\begin{aligned}
\mathcal{E} & :=\exp (\alpha|B| t), \\
C_{2} & :=\frac{|B|-v_{0}}{|B|+v_{0}}
\end{aligned}
$$

Note: if $t \rightarrow \infty$, then

$$
\begin{aligned}
& \alpha>0 \Rightarrow v(t) \rightarrow-|B| \Rightarrow z, B \text { become antiparallel } \\
& \alpha<0 \Rightarrow v(t) \rightarrow|B| \Rightarrow z, B \text { become parallel. }
\end{aligned}
$$

Substituting $v$ we can solve for $w$, which is a scalar function times a constant vector:

$$
\begin{aligned}
w(t) & =f(t) w(0), \\
f(t) & =\frac{\mathcal{E}\left(C_{2}+1\right)}{\mathcal{E}^{2} C_{2}+1} \longrightarrow 0 \text { as } t \rightarrow \infty, \text { if } \alpha \neq 0 .
\end{aligned}
$$

Substituting $w$ we can solve for $z$ :

$$
\begin{aligned}
z(t) & =\exp \left(g \hat{w}_{0}\right) z(0), \\
& =\cos \left(g\left|w_{0}\right|\right) z_{0}+\frac{\sin \left(g\left|w_{0}\right|\right)}{\left|w_{0}\right|} w_{0} \times z_{0},
\end{aligned}
$$

where

$$
\begin{aligned}
g \equiv g(t) & :=-\alpha \int_{0}^{t} f(\tau) d \tau=\frac{C^{2}+1}{|B| C}(\arctan C-\arctan (C \mathcal{E})) \\
C & :=\sqrt{C_{2}}=\sqrt{\frac{|B|-v_{0}}{|B|+v_{0}}}
\end{aligned}
$$


Note that the exp above is a matrix exponential, while the sin and cos are the usual scalar functions. Here $\exp \left(g \hat{w}_{0}\right)$ is expanded as a Magnus series [1]: the direction of $w(t)$ is constant, hence $g \hat{w}_{0}$ commutes with its integrals and Magnus series truncates after the first term. The evaluation of that term is by Rodriguez' formula, hence (23)

Evaluating $g$ numerically was a problem because eventually $v$ approaches $\pm|B|$ (physically this means $z$ becomes (anti-)parallel to $B$ ) so the $C$ in $g$ becomes zero. $g$ itself is not singular, however this presentation is difficult to evaluate. We used the following Taylor expansions in the implementation: if ||$B\left|-v_{0}\right|<0.0001$,

$$
g=-1+\mathcal{E}+C^{2}\left(-\frac{2}{3}+\mathcal{E}-\frac{1}{3} \mathcal{E}^{3}\right)+\mathcal{O}\left(C^{4}\right)
$$

and if ||$B\left|+v_{0}\right|<0.0001$,

$$
g=1-\mathcal{E}^{-1}+C^{-2}\left(\frac{2}{3}-\mathcal{E}^{-1}+\frac{1}{3} \mathcal{E}^{-3}\right)+\mathcal{O}\left(C^{-4}\right) .
$$

\section{A Lyapunov function}

Note that

$$
|w|^{2}=(z \times B) \cdot(z \times B)=-(B \times z) \cdot(z \times B)=-B \cdot z \times(z \times B)=\frac{-B}{\alpha} \cdot \dot{z}=-\frac{\dot{v}}{\alpha},
$$

hence

$$
\frac{d}{d t} v=-\alpha|z \times B|^{2} \leq 0, \text { if } \alpha \geq 0
$$

So $v$ is a Lyapunov function, when $\alpha$ is positive. If $H(z):=C v=C z \cdot B, C$ constant scalar, then

$$
\frac{d}{d t} H=-\alpha C|z \times B|^{2}
$$

that is, $H$ is a lyapunov function iff $\operatorname{sgn}(\alpha C)=1$. Later $\operatorname{sgn}(C)$ chooses between ferromagnet and antiferromagnet.

\section{Several spins}

Now we continue from (15), which can be written

$$
\dot{z}_{i j}=z_{i j} \times\left(-j_{K}\right) M \sum_{N N(i j)} z+\alpha z_{i j} \times z_{i j} \times\left(-j_{K}\right) M \sum_{N N(i j)} z .
$$

Recall from the previous discussion that $\alpha<0$ implies $z_{i j}$ tends to become parallel to

$$
\left(-j_{K}\right) M \sum_{N N(i j)} z
$$

This means, see (26), that if $j_{K}>0$, then $\operatorname{sgn}\left(\alpha j_{K}\right)=-1$ and energy $H$ is decreasing. In other words, for a ferromagnet negative $\alpha$ means energy damping.

To summarize, a ferromagnetic or antiferromagnetic spin system subject to Gilbert damping will uniformly dissipate energy for appropriate choice of the sign of the damping coefficient. Moreover, a Gilbert-damped system is spin-length conserving. 


\section{Numerical method}

To integrate, we split the vector field in even-odd way as in the conservative case (Section 2)

$$
\dot{z}_{i j}=V_{1}+V_{2}+V_{3}+V_{4},
$$

where

$$
\begin{aligned}
& V_{1}= \begin{cases}-j_{K} z_{i j} \times M \sum_{N N(i j)} z, & i+j \text { even } \\
0, & i+j \text { odd },\end{cases} \\
& V_{2}= \begin{cases}0, & i+j \text { even } \\
-j_{K} z_{i j} \times M \sum_{N N(i j)} z, & i+j \text { odd, }\end{cases} \\
& V_{3}= \begin{cases}-j_{K} \alpha z_{i j} \times z_{i j} \times M \sum_{N N(i j)} z, & i+j \text { even } \\
0, & i+j \text { odd },\end{cases} \\
& V_{4}= \begin{cases}0, & i+j \text { even } \\
-j_{K} \alpha z_{i j} \times z_{i j} \times M \sum_{N N(i j)} z, & i+j \text { odd } .\end{cases}
\end{aligned}
$$

Now, all these flows can be explicitly solved. For example $V_{1}$ : for $i+j$ odd $z_{i j}(t)=z_{i j}(0)$. For $i+j$ even, the sum over $N N(i j)$ includes only pairs $a, b$ with $a+b$ odd, hence they are constants (during $V_{1}$ ). Likewise in $V_{2}, V_{3}$, and $V_{4}$ the sums include only constants.

Hence in $V_{1}$ and $V_{2}$ we solve

$$
\dot{z}_{i j}=z_{i j} \times B, \quad B \text { constant },
$$

and in $V_{3}$ and $V_{4}$ we solve

$$
\dot{z}_{i j}=\alpha z_{i j} \times z_{i j} \times B, \quad B \text { constant },
$$

which are solved above. Note that (29), (30) have different $B$ 's. The implemented integrator is a symmetric composition of these exact flows:

$$
\hat{\Phi}_{t}:=\Phi_{4, \frac{t}{2}} \circ \Phi_{3, \frac{t}{2}} \circ \Phi_{2, \frac{t}{2}} \circ \Phi_{1, t} \circ \Phi_{2, \frac{t}{2}} \circ \Phi_{3, \frac{t}{2}} \circ \Phi_{4, \frac{t}{2}},
$$

where $\Phi_{i, t}=\exp \left(t V_{i}\right)$ are the exact flows.

An important feature of our method is that it dissipates energy when the flow (15) does. This can be seen in the following way: we solve the flows $V_{1}, \ldots, V_{4}$ exactly, hence every step in the composition (31) follows the energy of the associated vector field exactly. Now $\hat{\Phi}_{\Delta t}$ is a second order method and it follows the energy evolution with accuracy $O\left(\Delta t^{3}\right)$. With small enough time step $\Delta t$ the error is negligible and our method dissipates the energy.

\section{Thermostatted version}

The motivation behind using thermostats is keeping the system around some constant average temperature. This is a reasonable assumption for example in systems with heat baths. That is, we allow the energy to fluctuate. But, at the same time, we want to keep the spin lengths constant. This will be carried out by modifying the dissipation term introduced in previous sections. 
We use the thermostatting term suggested in 2]: choose a parameter $T$ (temperature) and compare the system's energy to it, allow the damping coefficient $\alpha$ to vary with time: $\alpha=\alpha(t)$ and

$$
\dot{\alpha}=-\left(\frac{\kappa}{\mathcal{N} T}\right)^{2} \sum_{i j}\left(\mathcal{I}-k T \nabla_{z_{i j}}\right) \cdot\left(z_{i j} \times z_{i j} \times \mathcal{I}\right),
$$

where $T$ is temperature, and $k$ is Boltzmann's constant which we hereafter take to be 1 . $\mathcal{N}$ is number of degrees of freedom, that is $\mathcal{N}=3 n^{2}$ since we have an $n \times n$ square lattice, $\kappa$ is coupling strength and typically $\approx \sqrt{\mathcal{N}}$. Here we take $\kappa / \mathcal{N}:=1 / n$. $\mathcal{I}$ is equal to:

$$
\mathcal{I} \equiv \nabla_{z_{i j}} H:=-j_{K} M \sum_{N N(i j)} z .
$$

The thermostatting variable $\alpha$ has been given the nickname "global demon" [2], so called due to its non-local (hence non-physical) character: it affects all spins simultaneously.

So our thermostatted system is

$$
\begin{aligned}
\dot{z}_{i j} & =z_{i j} \times \mathcal{I}+\alpha z_{i j} \times z_{i j} \times \mathcal{I} \\
\dot{\alpha} & =-\left(\frac{\kappa}{\mathcal{N} T}\right)^{2} \sum_{i j}\left(\mathcal{I}-T \nabla_{z_{i j}}\right) \cdot\left(z_{i j} \times z_{i j} \times \mathcal{I}\right) .
\end{aligned}
$$

It is possible to show that the ferromagnetic system thermostatted using (34), (355) samples from the canonical ensemble. This system also conserves spin length. Finally, one easily demonstrates that these equations are invariant under the simultaneous timecoordinate transformation $t \mapsto-t, z \mapsto-z, \alpha \mapsto-\alpha$, i.e. the equations are timereversible.

\section{Numerical method}

To integrate, we split the vector field in even-odd way as above, with the $\dot{\alpha}$ term:

$$
\left(\begin{array}{c}
\dot{z}_{i j} \\
\dot{\alpha}
\end{array}\right)=V_{1}+V_{2}+V_{3}+V_{4}+V_{5}
$$

where $V_{1}, V_{2}, V_{3}, V_{4}$ as in Section 3 (with $\alpha=\alpha_{0}=$ constant) and

$$
V_{5} \leftrightarrow\left\{\begin{array}{l}
z_{i j}=\text { constant } \\
\dot{\alpha}=\sum_{i j}\left(\left(z_{i j} \cdot B\right)^{2}-B \cdot B-2 T z_{i j} \cdot B\right) .
\end{array}\right.
$$

Here we have simplified:

$$
\begin{gathered}
B:=\mathcal{I}=-j_{K} M \sum_{N N(i j)} z=\text { indep. of } z_{i j}, \\
\nabla_{z} \cdot(z \times z \times B)=\nabla_{z} \cdot((z \cdot B) z-B)=2 z \cdot B, \\
B \cdot(z \times z \times B)=(z \cdot B)^{2}-B \cdot B .
\end{gathered}
$$

In $V_{5}$ all terms are constant so the equation with $V_{5}$ is trivially solved. But note that the update step of $\alpha$ is $O\left(n^{2}\right)$.

$$
\hat{\Phi}_{t}:=\Phi_{1, \frac{t}{2}} \circ \Phi_{2, \frac{t}{2}} \circ \Phi_{3, \frac{t}{2}} \circ \Phi_{4, \frac{t}{2}} \circ \Phi_{5, t} \circ \Phi_{4, \frac{t}{2}} \circ \Phi_{3, \frac{t}{2}} \circ \Phi_{2, \frac{t}{2}} \circ \Phi_{1, \frac{t}{2}},
$$


where $\Phi_{i, t}=\exp \left(t V_{i}\right)$ are the exact flows.

An important feature of this discretization is that it is time-reversible with respect to the mapping $z \mapsto-z, \quad \alpha \mapsto-\alpha, \quad t \mapsto-t$. This can be seen by recalling from Section 1.1 that if $f(R u)=-R f(u)$ for some linear involution $R$, then the Strang splitting gives a time-reversible map. Here $u:=(z, \alpha)$ and $R u:=(-z,-\alpha)$. Applying the rule four times in a row: first to $\Phi_{4}$ and $\Phi_{5}$ in the roles of $\Phi_{\tau, f_{1}}$ and $\Phi_{\tau, f_{2}}$ of Section 1.1. secondly to $\Phi_{3}$ and $\Phi_{4} \circ \Phi_{5}$ in a similar way, next to $\Phi_{2}$ and $\Phi_{3} \circ \Phi_{4} \circ \Phi_{5}$ and finally to $\Phi_{1}$ and $\Phi_{2} \circ \Phi_{3} \circ \Phi_{4} \circ \Phi_{5}$, we get the claim.

\section{Numerical results}

In all our simulations we used $n=50$, that is, a $50 \times 50$ lattice. We used ferromagnets with anisotropy: $D=\operatorname{diag}(1,1, \lambda)$. This is known as "easy plane" or "easy axis" anisotropy, corresponding to $\lambda<1$ or $\lambda>1$, respectively.

\subsection{Dissipated system}

Example 1. In Figure 1 we see evidence of the dissipation of energy. On the top part is energy in semilog scale, on the bottom part is the maximum norm of the discrete Laplacian during each time step. Here we used periodic boundary conditions, $\lambda=1.1, \alpha=-0.5$, and timestep $\Delta t=0.1$. The initial configuration was random (top left of Figure 2).

The evolution of the discrete Laplacian is understood as the system settling down to some formation, and this can be seen in Figure 2, which includes snapshots of the same simulation. The snapshots describe the $z$-components of the spins. The order of the pictures is top row first, from left to right. The darker a point is, the lower $z$-component it has. Black represents spin down, white spin up.

The result is typical: the dissipated system converges to two bands of up and down spins. We also tested zero boundary conditions, then the dissipated system typically converged to a single band.

\subsection{Thermostatted system}

Example 2. In Figures 3 and 4 is a thermostatted system with periodic boundaries, $\lambda=0.9, T=0.04$ and timestep $\Delta t=0.01$. The initial condition is random. In the top part of Figure 3 is the thermostatting variable $\alpha$ and in the middle part is the energy, and in the bottom part the maximum norm of the discrete Laplacian. After an initial phase both $\alpha$ and energy settle to an aperiodic oscillatory motion, $\alpha$ between -10 and +10 , energy between -4800 and -4300 . We plotted only 2000 steps but the behavior continued similarly for at least 25000 steps. In Figure 4 we can see slowly creeping boundaries; the reader is asked to compare the white areas. At $t=0.26$ it suddenly looks chaotic, then renders back to the creeping boundaries. This can be seen as a kind of stability of the creeping boundaries. After 25000 steps there still is slow motion, the system does not converge to any particular formation.

Example 3. Another example, in Figures [5],8] is a thermostatted system we call "wandering vortices". This beautiful system has random initial conditions, periodic boundaries, and parameters $\lambda=0.9, T=0.05$ and timestep $\Delta t=0.05$. Figures 5 and 6 represent the evolution of $\alpha$, energy, and averages of the energy over different time windows. Interestingly, the behavior of $\alpha$ is much wilder than in the Example 2. The snapshots in Figures 

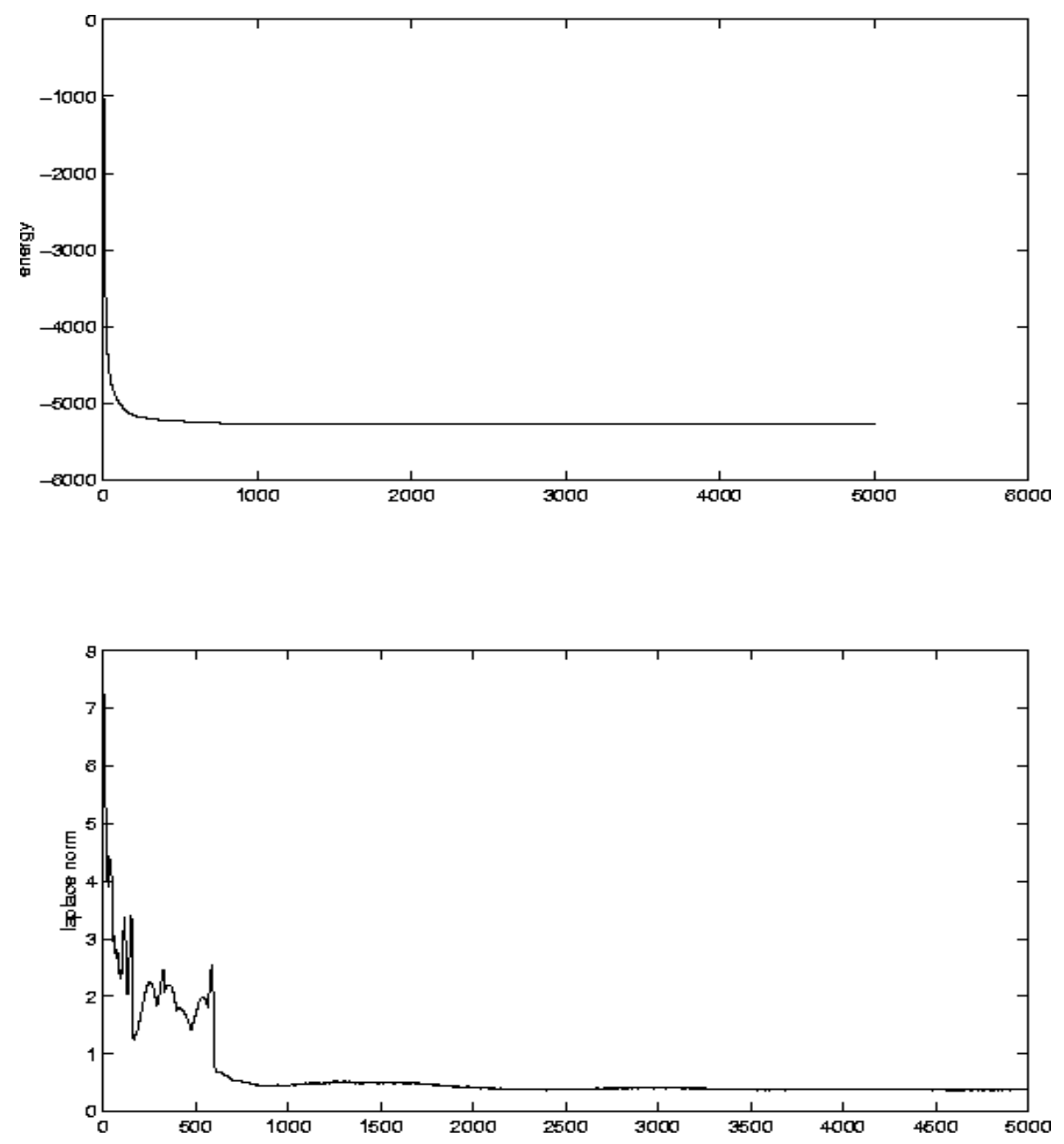

Figure 1: Energy of dissipated system.

78 show the $z$-components of the lattice. From random state, the system very quickly forms vortices on smooth surrounding, which move around for a short time, then look random again, then vortices again. Sometimes the vortices died out completely leaving us just with smooth surface, then reappeared again.

\section{Comparison to RK4 with projection}

For comparison we implemented the classical Runge-Kutta $4^{\text {th }}$ order method (RK4) with projection: after every step we normalize

$$
z_{i j, n e w}:=z_{i j, R K} /\left|z_{i j, R K}\right|
$$

where $z_{i j, R K}$ denotes the result of RK4 step. At very small timesteps for which the RK4 method could successfully integrate the problem, it was slightly faster than splitting, but the RK4 method became rapidly unstable as the stepsize and/or anisotropy were increased. The splitting method was able to handle large anisotropies $(\lambda=3)$ and step sizes $(\Delta t=0.3)$. However, we did not seek the limits of our splitting method. The values $\lambda=3$ and $\Delta t=0.3$ indicate the superior stability well enough at this stage.

We tested this projected RK4 on Examples 2 and 3. We kept the other parameters intact but changed the step size. As a sign of failure, we stopped the computation when 

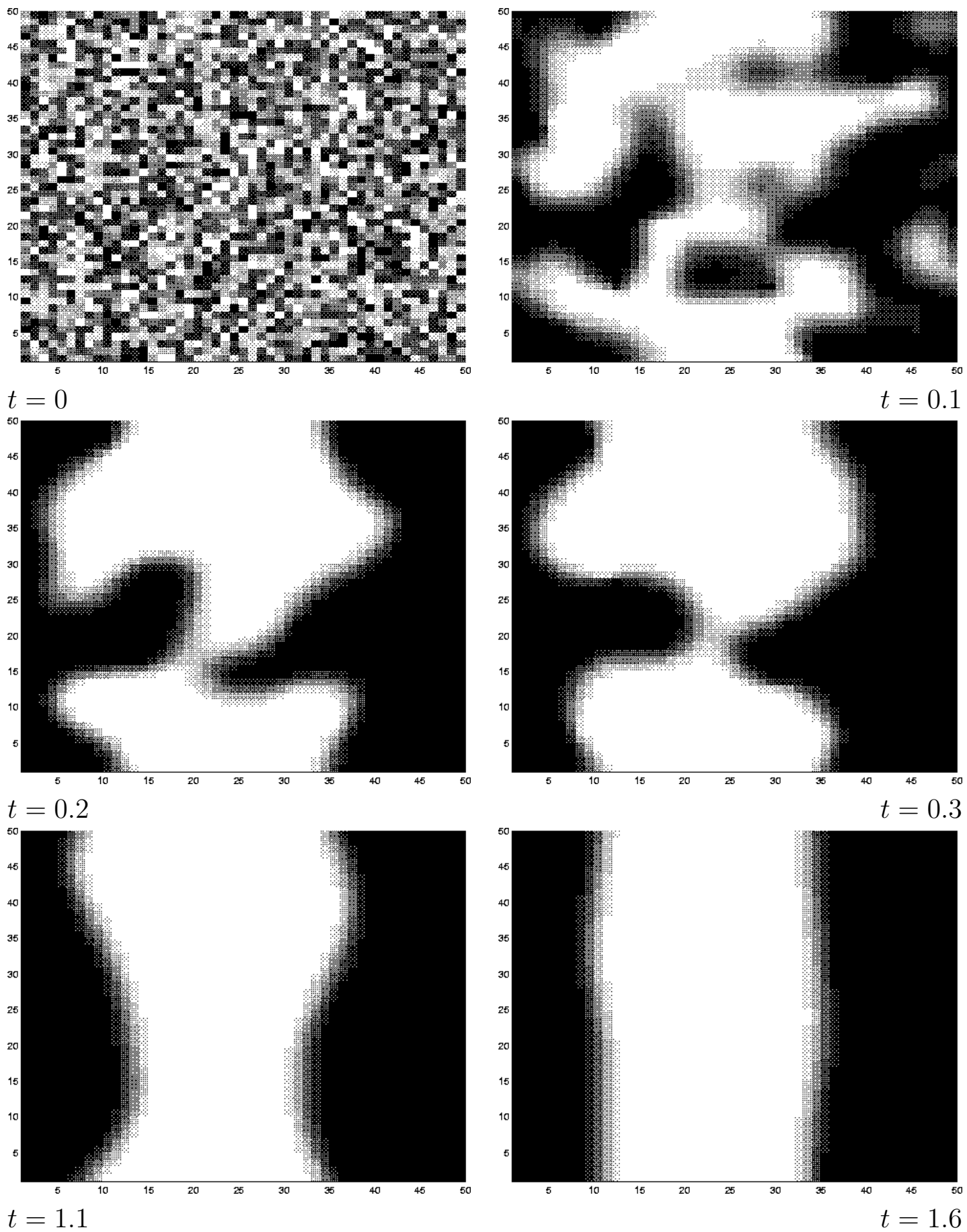

Figure 2: Snapshots of $z$-components (black is down spin, white is up spin) of the dissipated system, example 1. 

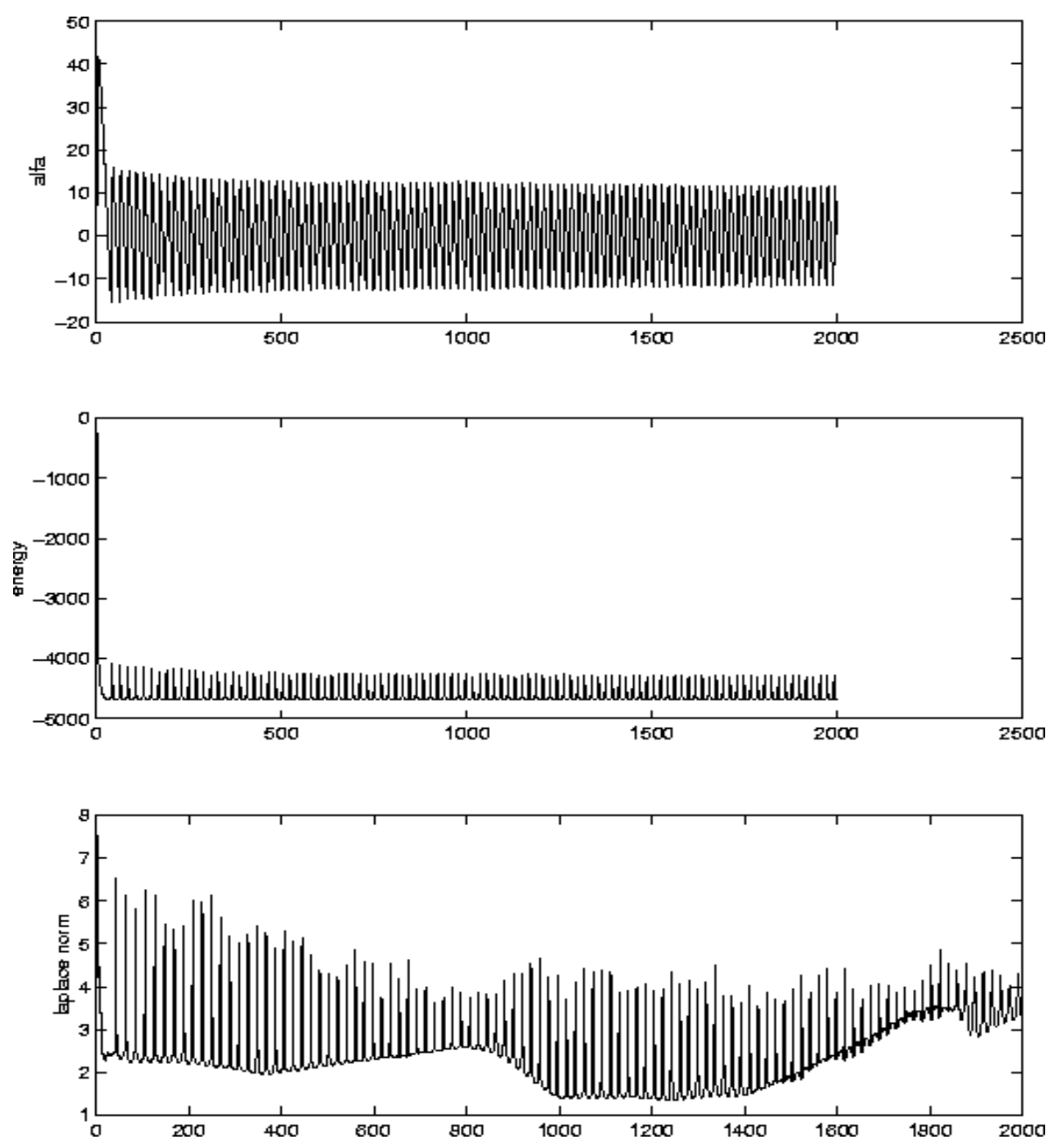

Figure 3: Energy, alpha, and norm of Laplacian of thermostatted system.

the code started to produce infinities. In Example 2 the maximal timestep was 0.01, while in Example $3 \Delta t_{\max }=0.015$. The results are summarized in table 1.

\section{Discussion}

In this paper we have developed and tested a geometric integrator for a semi-discretized Landau-Lifshitz-Gilbert (LLG) equation which includes a nonlinear dissipative term, as well as for a more complicated thermostatted model, following the approach of Bulgac and Kusnetsov.

The integrator for the dissipated system is shown to have a dissipative property. However, it is difficult to compare since we do not know the exact continuous solution. LLG is currently a very active topic of research, see more details in the introduction. However, it seems that so far there has not been developed a geometric integrator for a thermostatted system.

Trying to simulate the thermostatted systems with projected RK4 revealed both the features of a stiff ODE and features of a conservative system. The combination is extremely difficult for standard form numerical methods. The key feature of our splitting method is that it is constructed from composition of building blocks that simulate each of the two components of the system correctly. 

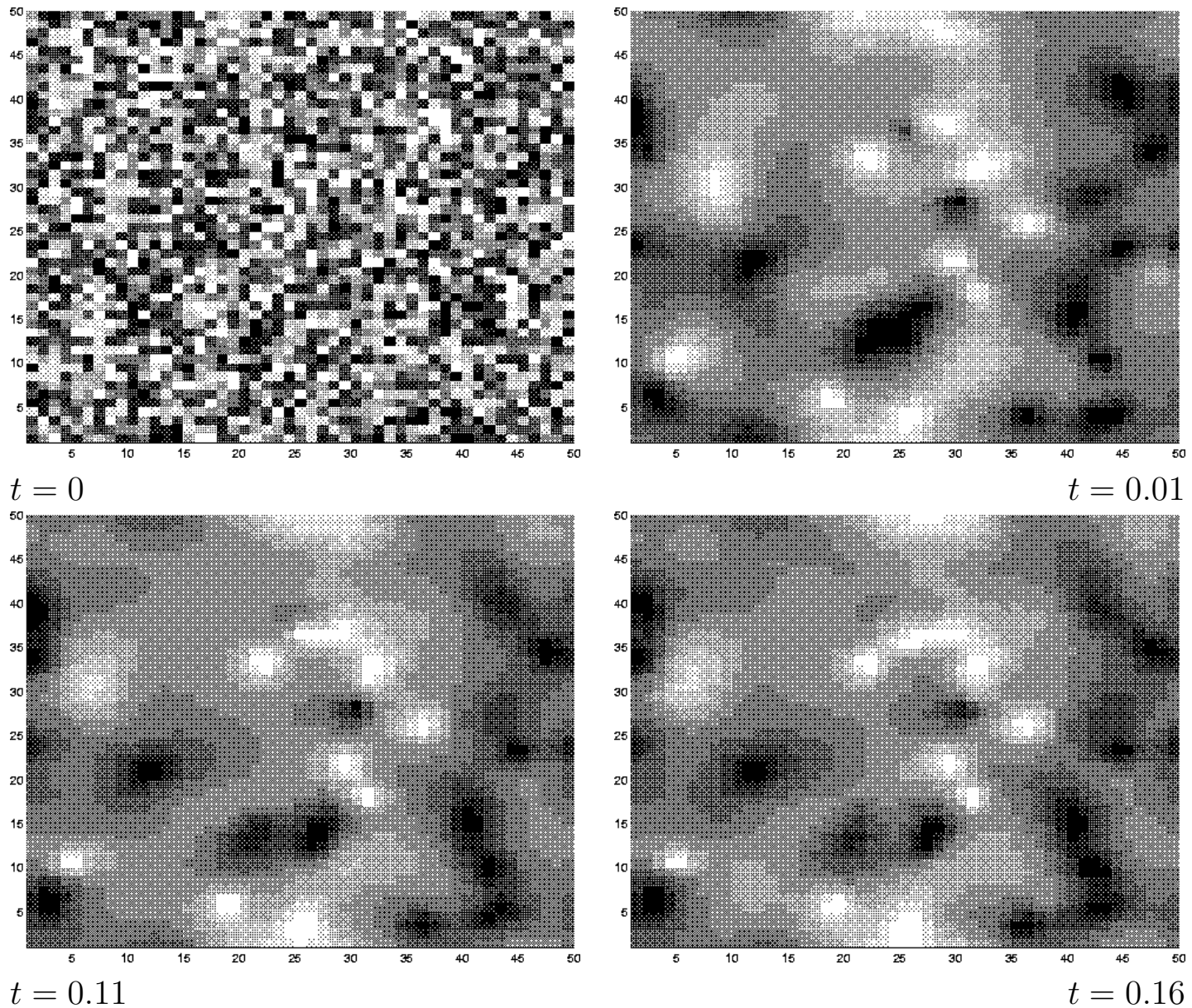

$t=0.11$
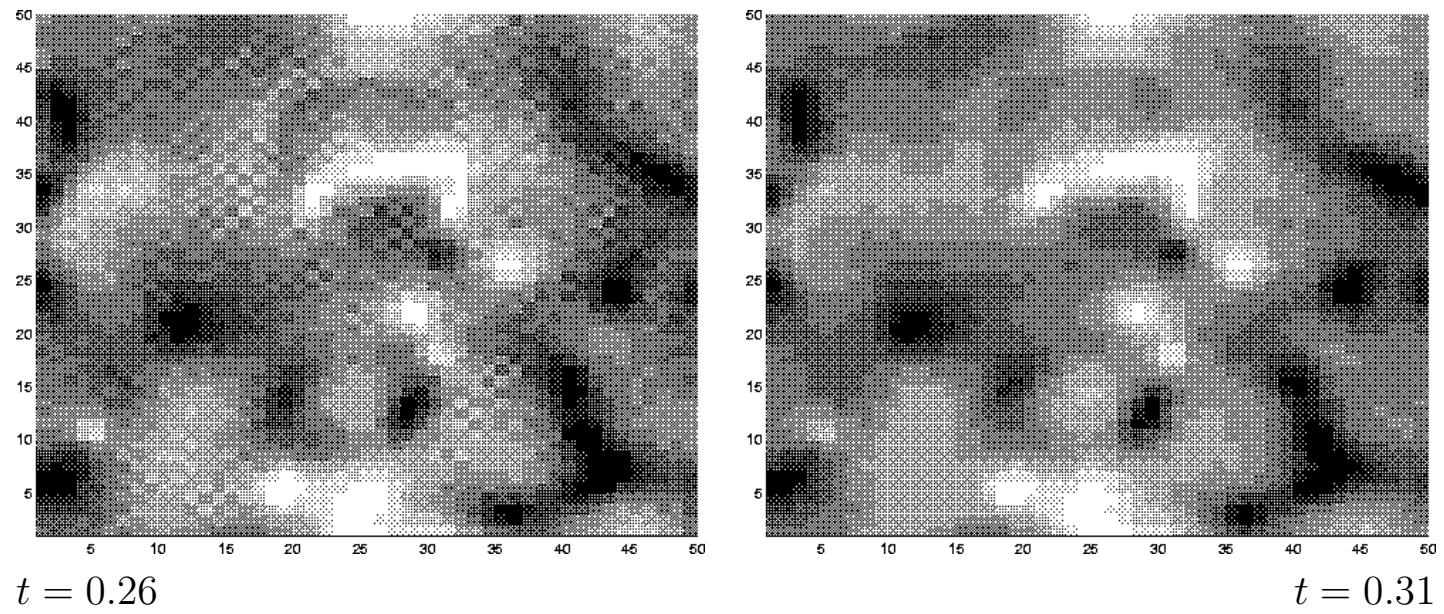

Figure 4: Snapshots of $z$-components (black is down spin, white is up spin) of thermostatted system, Example 2. 

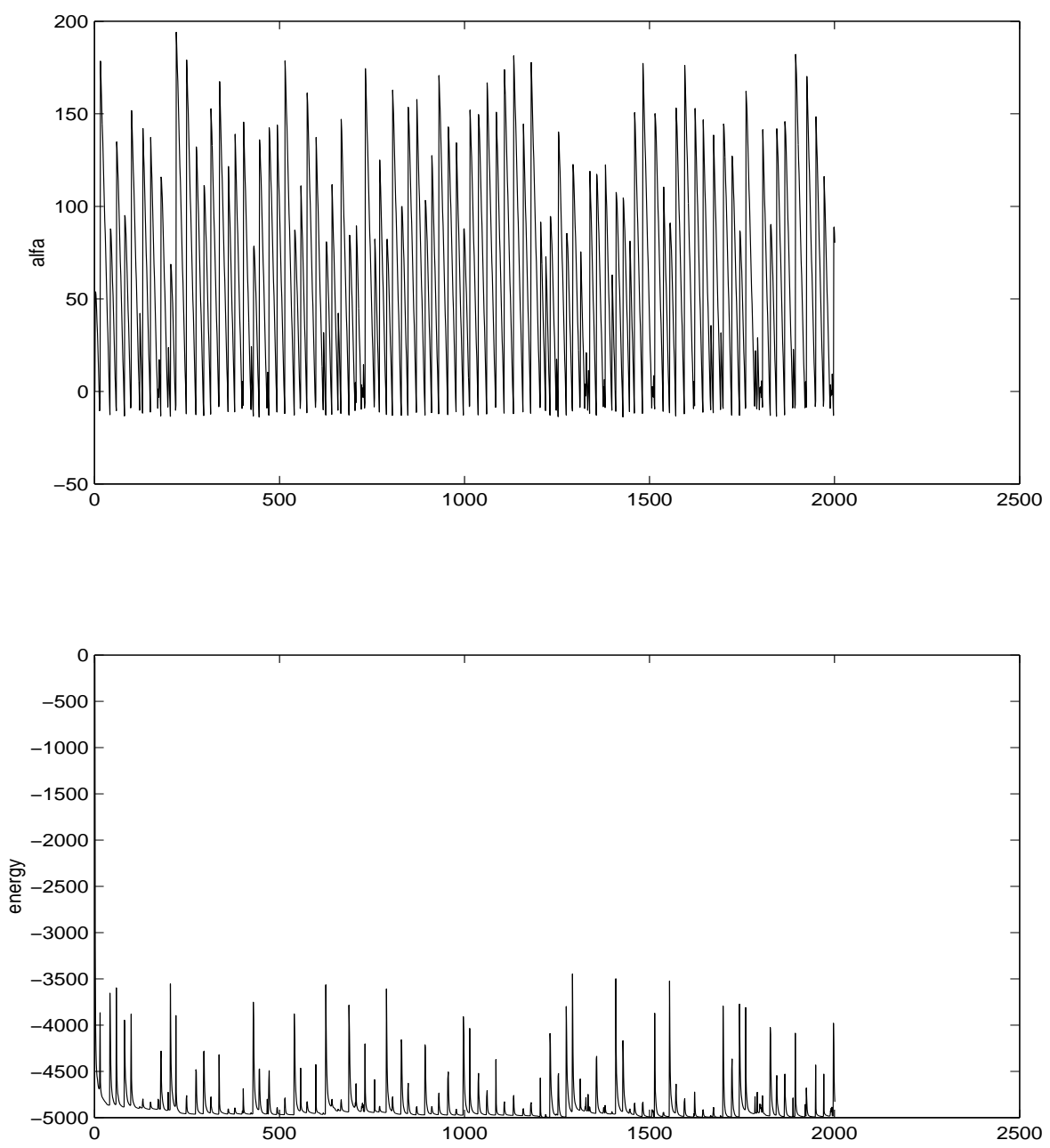

Figure 5: Energy and alpha of "wandering vortices", Example 3.

Simulation with our new thermostatted method has revealed interesting phenomena: slowly creeping boundaries, or wandering vortices, both of which appear from random initial conditions. Our informal term "wandering vortices" in Example 3 refers not to certain particular vortices that survive throughout the whole simulation, but to a situation where we have two or more vortices which wander for a while, then violently crash and form new vortices. Intermediate states include "quasi-chaotic" state, an informal term by which we mean a state that suddenly appears and looks random but is not, since it renders immediately back to (almost) the same smooth motion.

The RK4 method is less stable. The stepsize restriction is an order of magnitude smaller compared to our splitting method. This is evidence of stiffness in the ODEs, and a better choice might seem to be a stiff solver on this account, but if one uses a stiff solver the result would be poor resolution of the conservative evolution which is also an important component of the dynamics of the system. The best compromise is therefore a composition scheme, such as that outlined here, which separately and appropriately resolves each term of the system.

We anticipate that this work will stimulate further research in the development of thermostatted numerical methods for systems with complicated nonlinear structure."

Acknowledgement. The authors are grateful to Jason Frank both for valuable comments and providing the code of [10]. The author TA was supported by the Academy of Finland. 

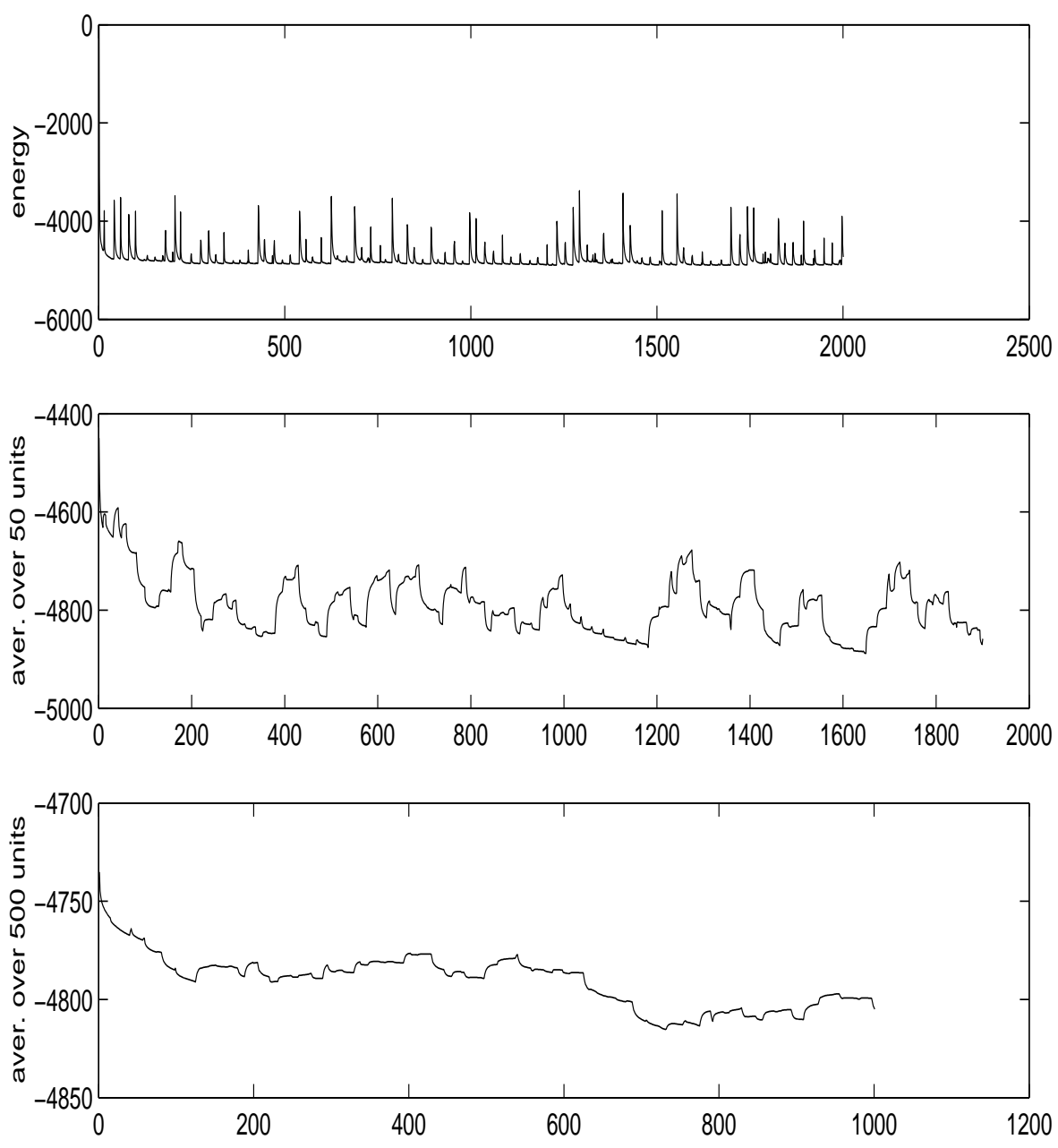

Figure 6: Averages of energy of "wandering vortices", Example 3.

BL was supported by the Engineering and Physical Sciences Research Council, grant GR/R03259/01.

\section{References}

[1] J.C. Anderson. Magnetism and Magnetic Materials. Chapman and Hall, 1968.

[2] V.P. Antropov, M.I. Katsnelson, B.N. Harmon, M. van Schilfgaarde, and D. Kusnezov. Spin dynamics in magnets: equation of motion and finite temperature effects. Phys. Rev. B, 54(2):1019-1035, 1996.

[3] E. Barth, B. Laird, and B. Leimkuhler. Generating generalized distributions from dynamical simulation. J. Chem. Phys., 118, 5759-5768, 2003.

[4] S. Bond, B. Laird, and B. Leimkuhler. The Nose-Poincare method for constant temperature molecular dynamics. J. Comput. Phys., 151, 114-134, 1999.

[5] A. Bulgac and D. Kusnezov. Canonical ensemble averages from pseudomicrocanonical dynamics. Phys. Rev. A, 42(8):5045-5048, 1990. 

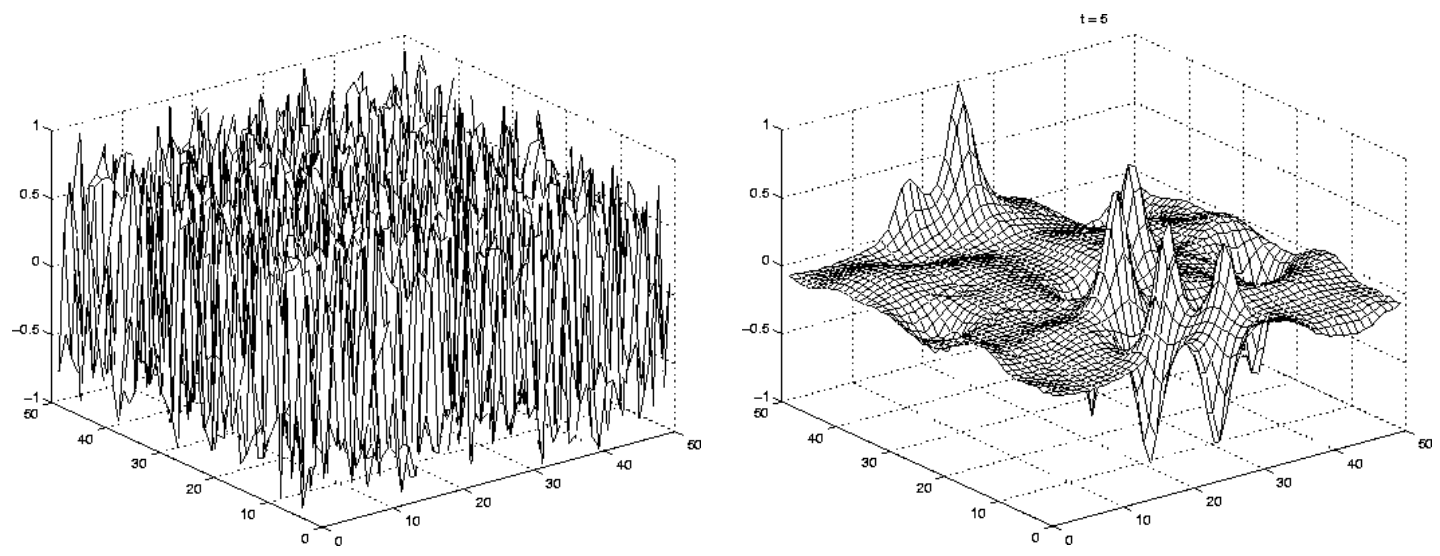

$t=0$
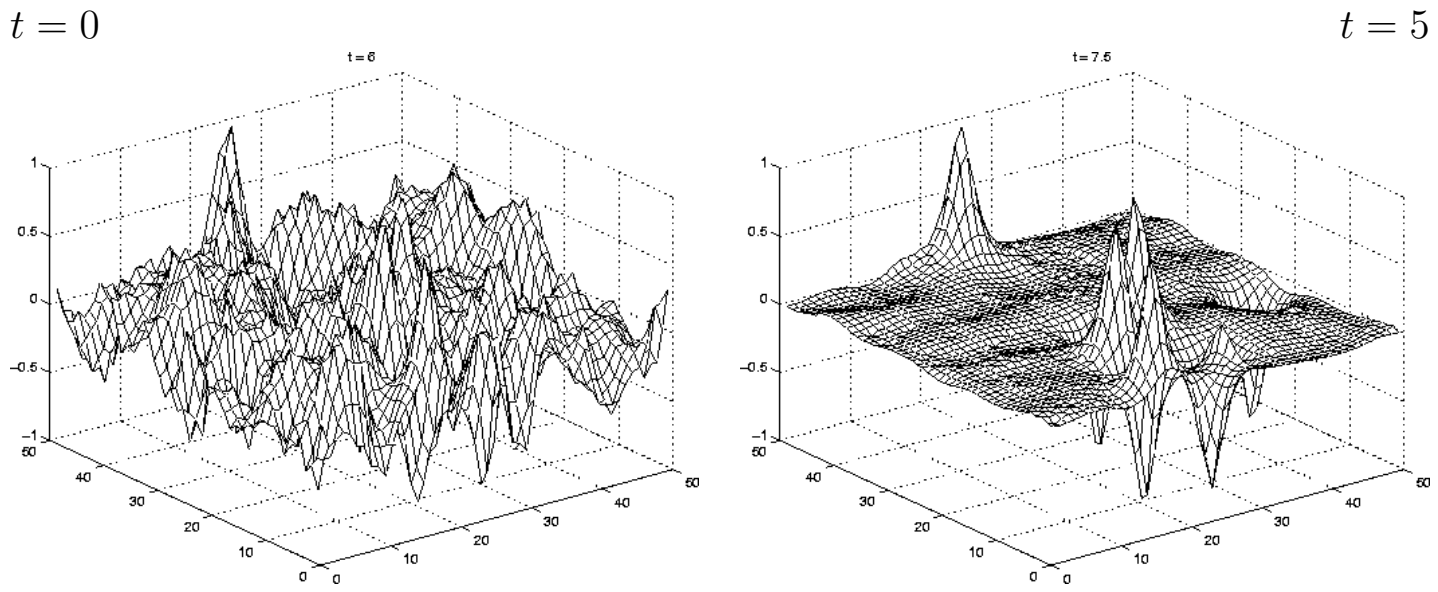

$t=6$
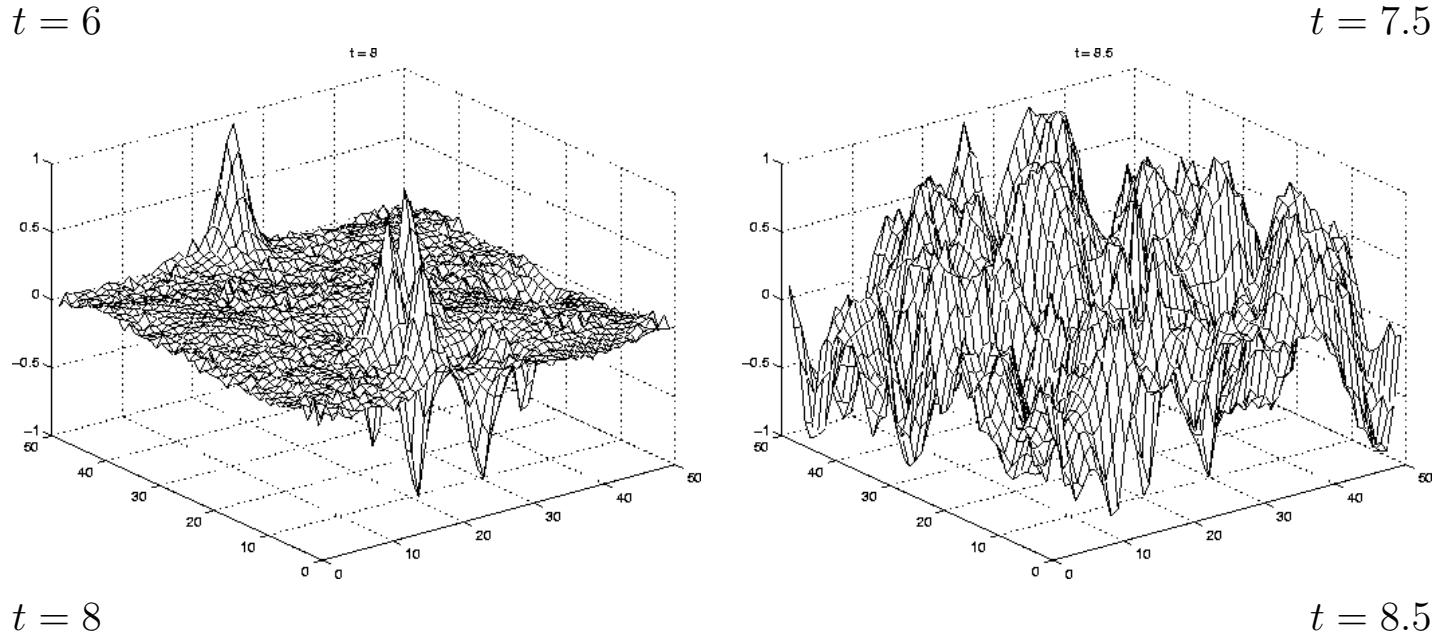

$t=8$

Figure 7: Snapshots of "wandering vortices", Example 3. 

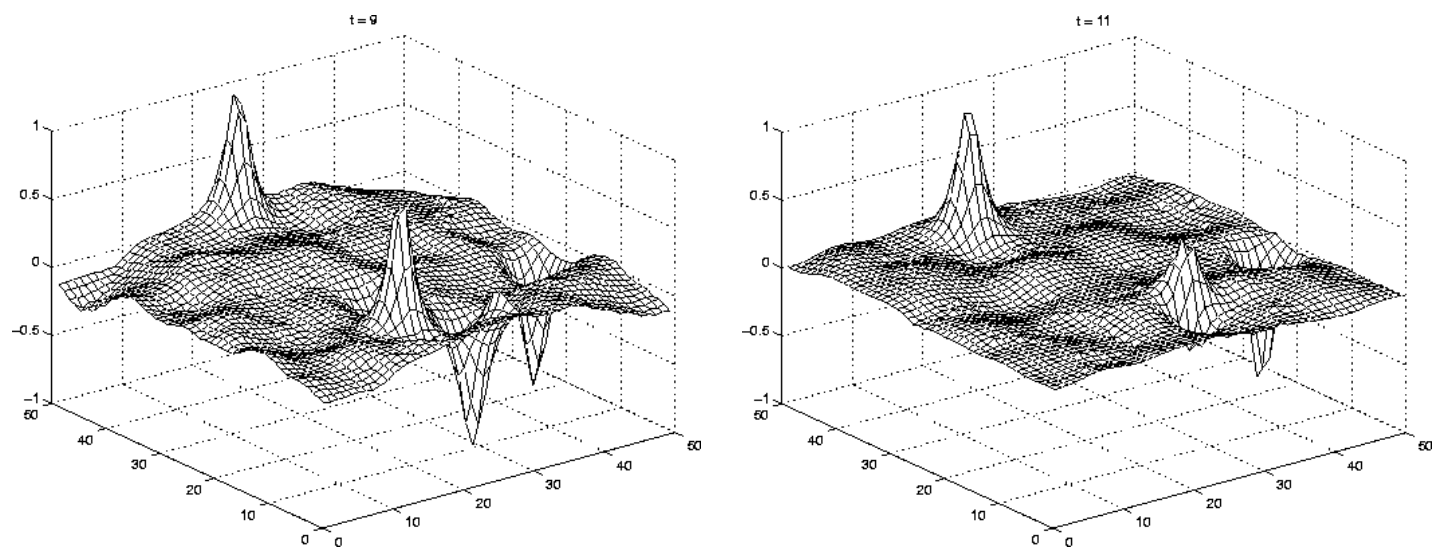

$t=9$
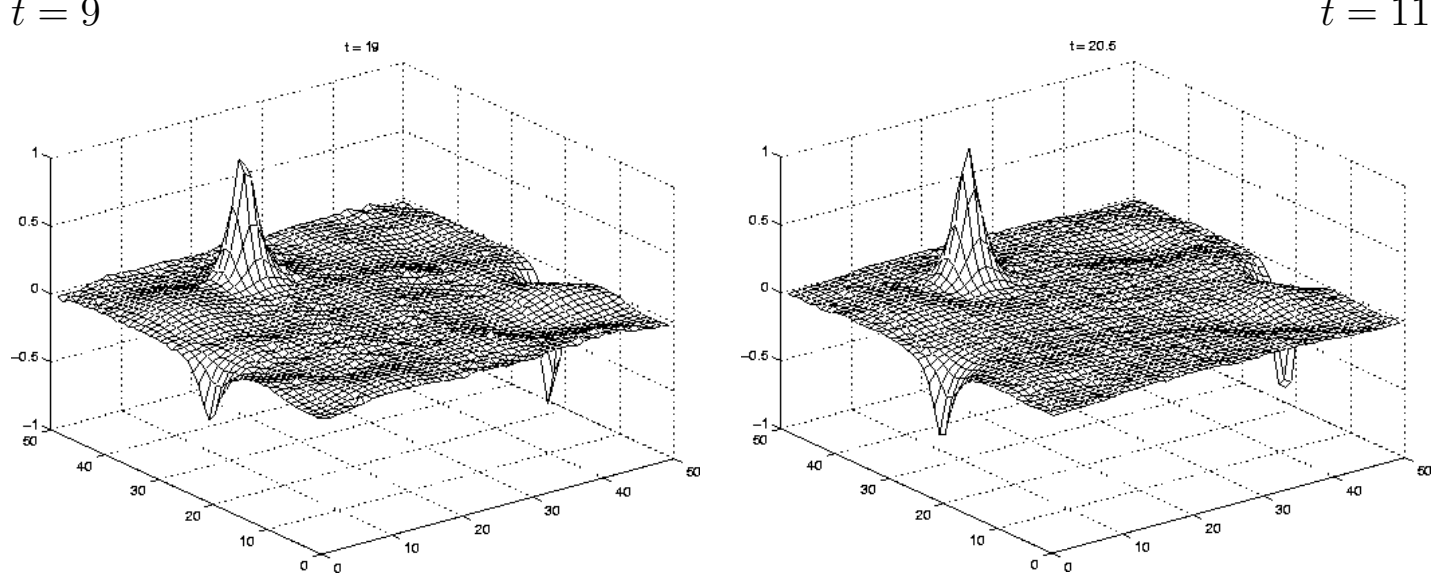

$t=19$

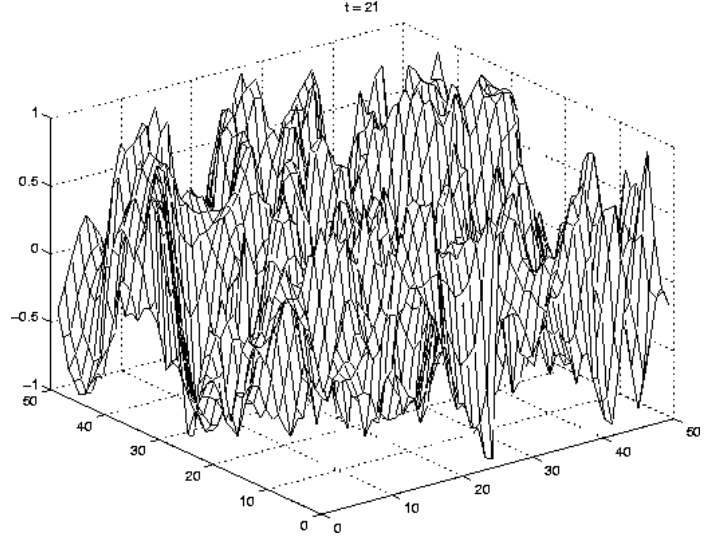

$t=21$
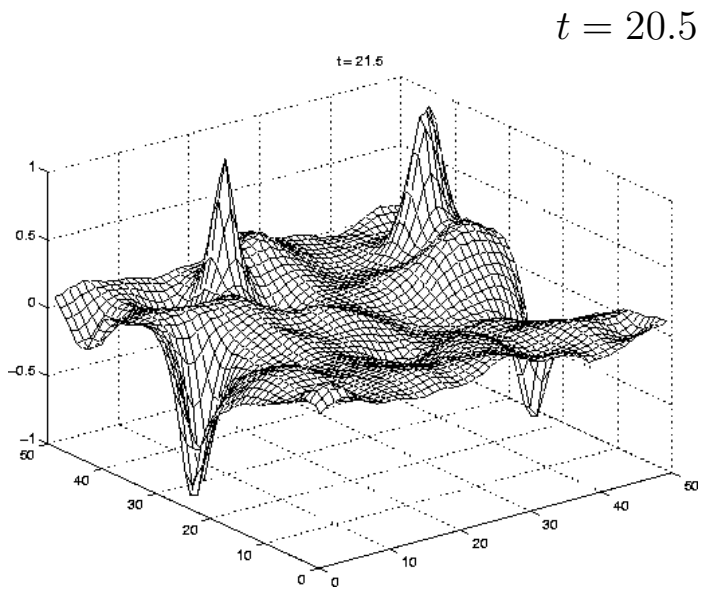

$t=21.5$

Figure 8: Snapshots of "wandering vortices", Example 3. 

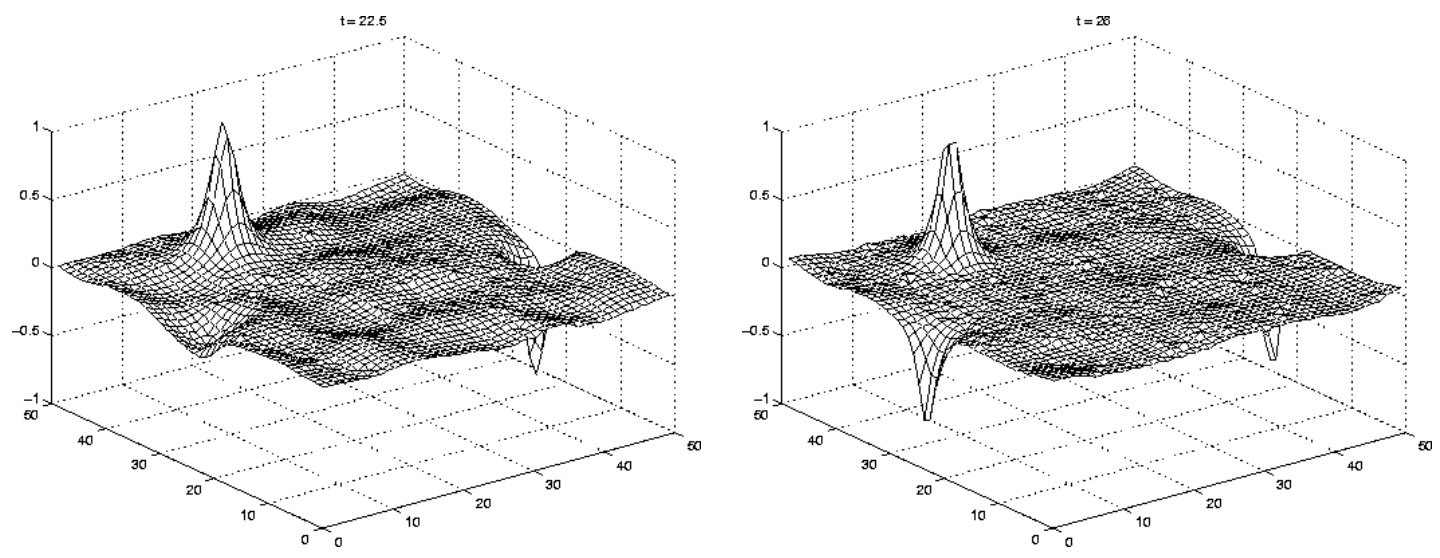

$t=22.5$
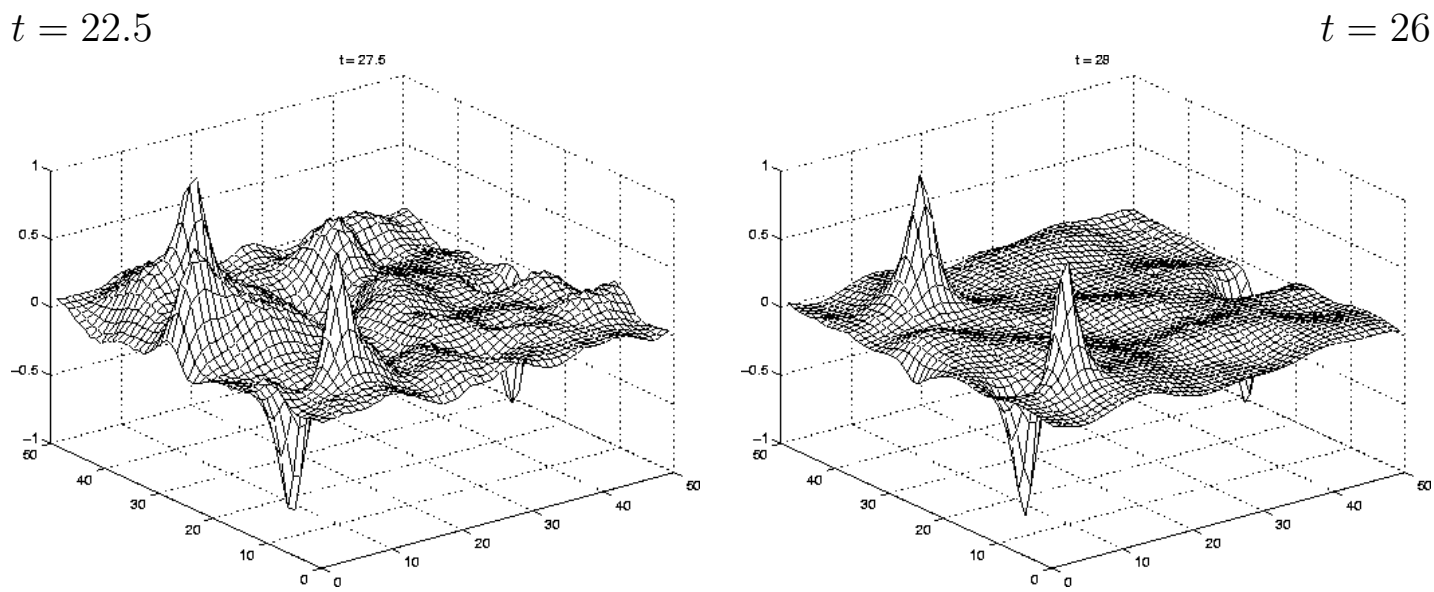

$t=27.5$
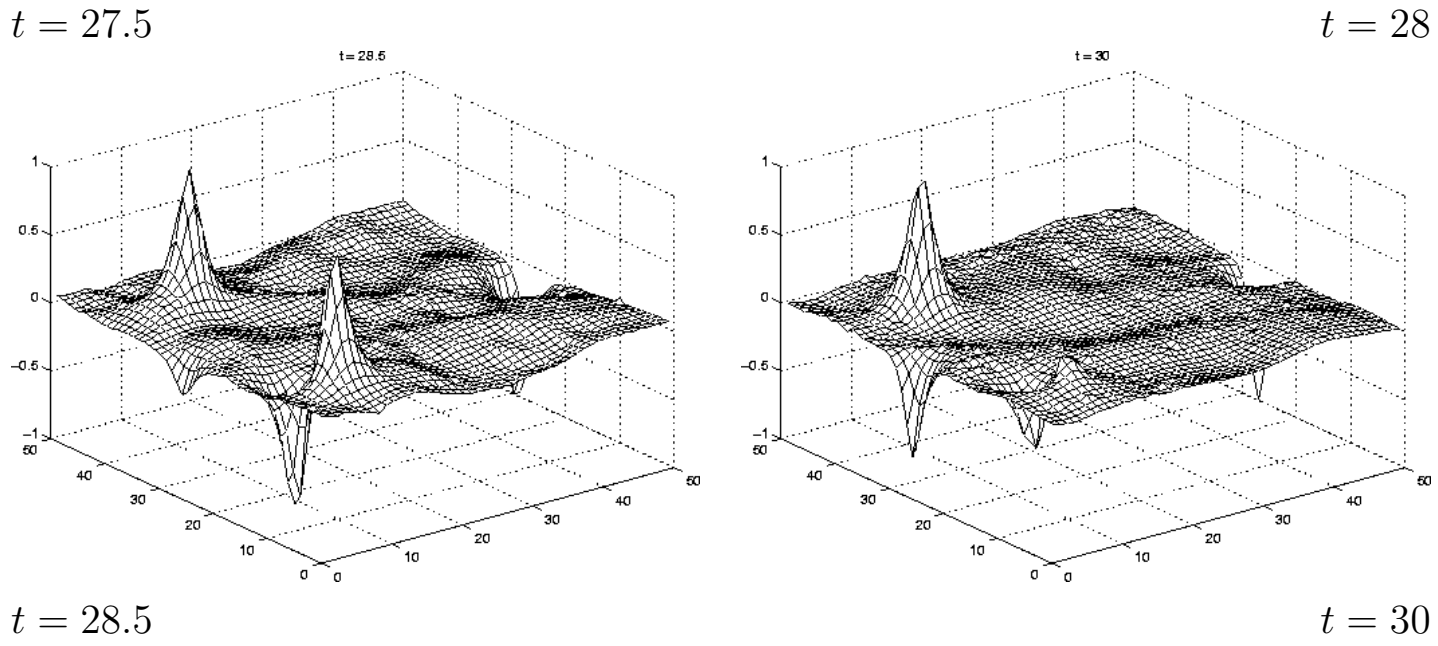

Figure 9: Snapshots of "wandering vortices", Example 3. 


\begin{tabular}{|l|c|c|}
\hline Example 2 & $\Delta t$ & RK4 survived until $t=$ \\
& 0.020 & 0.060 \\
& 0.015 & 0.075 \\
& 0.012 & 0.084 \\
& 0.011 & 0.099 \\
& 0.0105 & 0.116 \\
& 0.0102 & 0.235 \\
& 0.010 & $>200$ \\
\hline Example 3 & $\Delta t$ & RK4 survived until $t=$ \\
& 0.020 & 0.080 \\
& 0.019 & 0.095 \\
& 0.017 & 0.102 \\
& 0.016 & 0.112 \\
& 0.015 & $>150$ \\
\hline
\end{tabular}

Table 1: Failure of projected RK4

[6] W. E and X.-P. Wang. Numerical methods for the Landau-Lifshitz equation. SIAM J. Num. Anal., 38(5):1647-1665, 2000.

[7] L.D. Faddeev and L.A. Takhtajan. Hamiltonian Methods in the Theory of Solitons. Springer Series in Soviet Mathematics. Springer, 1987.

[8] X. Feng and P.B. Visscher. Stability and accuracy of Euler and quaternion micromagnetic algorithms. J. Appl. Phys., 91(10):8712-8714, 2002.

[9] J. Frank. Geometric space-time integration of ferromagnetic materials. Technical report, CWI, Amsterdam, the Netherlands, October 2003.

[10] J. Frank, W. Huang, and B. Leimkuhler. Geometric integrators for classical spin systems. J. Computat. Phys., 133, 1997.

[11] E. Hairer, C. Lubich, and G. Wanner. Geometric Numerical Integration, volume 31 of Springer Series in Computational Mathematics. Springer, 2002.

[12] B. Laird and B. Leimkuhler. Generalized dynamical thermostatting technique. Phys. Rev. E, 68, art. 016704, 2003.

[13] B. Laird and J. Sturgeon. Symplectic algorithm for constant-pressure moleculardynamics using a Nose-Poincare thermostat. J. Chem. Phys. 112 , 3474-3482, 2000 .

[14] D.P. Landau, A. Bunker, H.G. Evertz, M. Krech, and S.-H. Tsai. Spin dynamics simulations - a powerful method for the study of critical dynamics. arXiv:cond-mat 9912375, Center for Simul. Phys., Univ. Georgia, USA, Dec 1999.

[15] B. Leimkuhler. A separated form of Nosé dynamics for constant temperature and pressure simulation. Comput. Phys. Comm., 148, 206-213, 2002.

[16] B. Leimkuhler and S. Reich. Geometric Integrators in Hamiltonian Mechanics. Cambridge University Press, 2004. 
[17] D. Lewis and N. Nigam. Geometric integration on spheres and some interesting applications. J. Comp. Appl. Math., 151:141-170, 2003.

[18] R. McLachlan and R. Quispel. Splitting methods. Acta Numerica, 11:341-434, 2002.

[19] A. Prohl. Computational Micromagnetism. Adv. Numer. Math. Teubner, Stuttgart, 2001.

[20] J. M. Sanz-Serna and M. P. Calvo. Numerical Hamiltonian Problems. Chapman \& Hall, 1994.

[21] M. Slodička and I. Cimrák. Numerical study of nonlinear ferromagnetic materials. Appl. Numer. Math., 46:95-111, 2003.

[22] P.B. Visscher and X. Feng. Quaternion-based algorithm for micromagnetics. Phys. Rev. B, 65:104412, 2002.

[23] X.-P. Wang, C.J. García-Cervera, and W. E. A Gauss-Seidel projection method for micromagnetics simulations. J. Computat. Phys., 171:357-372, 2001.

[24] H. Yoshida. Construction of higher order symplectic integrators. Phys. Lett. A, 150(5-7):262-268, 1990.

\section{Appendix: Lie-Poisson Canonical Sampling Technique}

Consider first the Poisson rigid body system consisting of a Hamiltonian $H(z)$ together with structure matrix $J(z)$ admitting Casimir $|z|$. Based partly on [12] we construct an augmented Hamiltonian with additional variables $\sigma, \pi_{\sigma}, \theta, \pi_{\theta}$ :

$$
\tilde{H}=H(Q(\theta) z)+\pi_{\sigma}^{2} / 2 \mu+k T \ln \sigma+G\left(\theta, \pi_{\theta}, \pi_{\sigma}\right) .
$$

where $Q$ is an orthogonal matrix depending on parameter(s) $\theta$. The Lie-Poisson structure is just the rigid body Poisson structure augmented by the canonical structure for the augmenting variables. Under assumption of ergodicity, and some very mild technical conditions similar to those obtained in [12, this Hamiltonian can be shown to provide canonical sampling from microcanonical trajectories i.e.

$$
\iiint \int f(Q(\theta) z) \delta[\tilde{H}-E] d \sigma d \theta d \pi_{\sigma} d \pi_{\theta}=f(z) \exp \left(-\frac{1}{k T} H(z)\right),
$$

with preservation of the Casimir due to $Q$ being orthogonal. In order to obtain ergodicity, the "bath Hamiltonian" $G$ should be sufficiently complicated.

In the case of a spin system, $H\left(z_{1}, z_{2}, \ldots, z_{N}\right)$, we may introduce a separate unit 3 -vector $\theta_{i}$ for each spin vector. Then the Hamiltonian

$$
\tilde{H}=H\left(Q\left(\theta_{1}\right) z_{1}, Q\left(\theta_{2}\right) z_{2}, \ldots, Q\left(\theta_{N}\right) z_{N}\right)+\pi_{\sigma}^{2} / 2 \mu+k T \ln \sigma+G\left(\theta, \pi_{\theta}, \pi_{\sigma}\right),
$$

will enable canonical sampling. For example $Q(u)$ can be a Householder transformation,

$$
Q(u)=I-2 \frac{u u^{T}}{u^{T} u}
$$

and the bath Hamiltonian $G$ can describe a coupled system of spherical pendula involving in some nontrivial way the parameter $\pi_{\sigma}$. 